\title{
P9: Der Transparenzbericht als Entscheidungshilfe für die Wahl der Pflegeeinrichtung
}

\author{
Franziska Götz • Maria Machansky • Carola Oehme • \\ Jennifer Thomas · Julia Voigt • Andreas Wahl • \\ Tina Zeun
}

Online publiziert: 31. Oktober 2013

(C) Springer-Verlag Wien 2013

Einleitung/Fragestellung: Seit 2009 werden jährlich die erbrachten Leistungen von Pflegeeinrichtungen und deren Qualität überprüft. Dabei beinhaltet die Pflegetransparenzvereinbarung für den stationären Bereich 64 bewertungsrelevante Kriterien, die sich wiederum auf vier Qualitätsbereiche aufteilen. Ziel unserer Studie war es, herauszufinden, ob zwischen den einzelnen Qualitätsbereichen Unterschiede auftreten.

Methodik/Stichprobe: Die Untersuchung umfasste Transparenzberichte von 353 stationären Pflegeeinrichtungen ohne fachlichen Schwerpunkt in Sachsen (Stichtag: 15.06.13, Quelle: www.pflegelotse.de). Dabei wurden deskriptive Statistiken und Häufigkeiten für die Qualitätskriterien berechnet. Des Weiteren wurden anhand des Chi-Quadrat-Tests Zusammenhänge zwischen den Bewertungen, Einrichtungsmerkmalen und dem Prüfdatum untersucht $(P<0,05)$.

Ergebnisse: Die durchschnittliche Gesamtnote für die 64 bewertungsrelevanten Transparenzkriterien betrug für alle Einrichtungen 1,11. Es zeigte sich bei der Datenauswertung der Bewertungskriterien eine Spannweite der Noten von 1,0 bis 3,4. Der Qualitätsbereich „Pflege und medizinische Versorgung“ erreichte mit 1,24 im Vergleich zu den übrigen
Bereichen die geringste Durchschnittsnote. Das Qualitätskriterium mit der niedrigsten Bewertung war die Durchführung der Kontrakturenprophylaxen $(1,93)$. Ferner waren es Kriterien, wie die Durchführung der Dekubitusprophylaxen $(1,86)$ und die entsprechend den Bedürfnissen und Gewohnheiten des Bewohners durchgeführte Körperpflege $(1,46)$, welche die geringe Durchschnittsnote verursacht haben. Am höchsten wurde der Qualitätsbereich ,Soziale Betreuung und Alltagsgestaltung“" mit der durchschnittlichen Note 1,02 bewertet. Zwischen der Bewohneranzahl, dem Jahr der Prüfung, dem Trägerstatus sowie Gesamtbewertung bzw. den vier Qualitätsbereichen konnten wir keine statistisch signifikanten Zusammenhänge feststellen.

Diskussion/Schlussfolgerungen: Basierend auf den Ergebnissen sind Tendenzen erkennbar, dass sich Pflegeeinrichtungen vor allem auf die Bereiche Alltagsgestaltung, Wohnen und Hauswirtschaft konzentrieren, um die Ergebnisse der Qualitätsprüfungen positiv zu beeinflussen. Aufgrund der daraus resultierenden durchgehend positiven Gesamtbewertungen aller untersuchten Pflegeheime, kommen wir zu dem Ergebnis, dass sich Transparenzberichte als alleiniges Auswahlkriterium für die Wahl einer Pflegeeinrichtung nur bedingt eignen.

F. Götz $\cdot$ M. Machansky $\cdot$ C. Oehme $\cdot$ J. Thomas $\cdot$ J. Voigt $\cdot$

A. Wahl $\cdot$ T. Zeun

Fakultät Gesundheits- und Pflegewissenschaften,

Westsächsische Hochschule Zwickau, Zwickau, Deutschland 